\section{Brain, Behavior \\ and Evolution}

Ashwell, K.W.S. 223

Ball, G.F. 119 (A)

Beisel, K.W. 182

Cheroske, A.G. 116 (A)

Cronin, T.W. 116 (A)

Evans, A.K. 116 (A)

Fernald, R.D. 141

Fraser, E. 119 (A)

Fritzsch, B. 182

Fuxe, K. 42

Geovannini, H. 61

Gibbs, M.A. 70, 163

Goodson, J.L. 116 (A)

Grabul, D.S. 118 (A)

Green, R.L. 85

Gruberg, E.R. 11

Gutiérrez-Ospina, G. 61

Hardman, C. 223

Hernández-Echeagaray, E. 61

Hillis, D. 120 (A)

Hoedl, W. 118 (A)

Hofmann, H.A. 119 (A)

Hoke, K.L. 116 (A)

Holmes, P.H. 1

Imura, K. 242

Ito, H. 120 (A), 242

Iwaniuk, A.N. 117 (A)

$(\mathrm{A})=$ Abstracts

Jeffery, W.R. 120 (A)

Keifer, J. 207

Kimmel, C.B. 118 (A)

Krumlauf, R. 115 (A)

Lacalli, T.C. 148

Lorenzo, D. 104

Lu, Y. 120 (A)

McNeil, R. 19

Manger, P.R. 42

Marín, G. 19

Maves, L. 118 (A)

Mitchell, M. 19

Narins, P.M. 118 (A)

Northcutt, M.S. 125

Northcutt, R.G. 1, 70, 118 (A), 125, 198

O’Bryant, E.L. 34

Padilla, P. 61

Paxinos, G. 223

Plassmann, W. 1

Ramírez, Y. 19

Renn, S.C.P. 119 (A)

Ridgway, S.H. 42

Rojas, L.M. 19

Rose, G.J. 85

Rosen, G.J. 34
Saidel, W.M. 1

Saltzman, H. 11

Sánchez, N. 61

Sartor, J.J. 119 (A)

Siegel, J.M. 42

Soares, D. 120 (A)

Soma, K.K. 118 (A)

Swender, D. 34

Uribe-Querol, E. 61

Velluti, J.C. 104

Wade, J. 34

Whitlock, K.E. 126

Wylie, D.R.W. 117 (A)

Xue, H.-G. 242

Yamamoto, N. 120 (A), 242

Yang, C.-Y. 242

Zacharatos, M. 11

Zakon, H.H. 120 (A)

Zhu, D. 207

Zwickl, D. 120 (A) 